\title{
Providing Reproductive Health Module Within Hiv Care And Treatment Centre In Kenya
}

\author{
Mosol Priscah, ${ }^{1}$ Kosgei J Rose, ${ }^{2}$ Mabeya Hillary, ${ }^{3,4,5}$ Sitienei Jackline, ${ }^{3,}{ }^{6}$ Sum \\ Tecla, ${ }^{4}$ Chelagat Dinah, ${ }^{7}$ Namaloba Ruth, ${ }_{4,10}^{8}$ Braitstein Paula, ${ }^{3,9}$ Siika Abraham ${ }^{3,}$ \\ ${ }^{1}$ Department of Nursing, Egerton University, Kenya \\ ${ }^{2}$ University of Nairobi, School of medicine, Department of Obstetrics and Gynaecology, Nairobi, Kenya \\ ${ }_{3}^{3}$ Academic Model Providing Access To Healthcare (AMPATH), Eldoret, Kenya \\ ${ }^{4}$ Moi Teaching and Referral Hospital, Kenya \\ ${ }^{5}$ Moi University, School of Medicine, Department of Obstetrics and Gynaecology, Eldoret, Kenya \\ ${ }^{6}$ Moi University, School of Public Health, Department of Health Policy and Management, Eldoret, Kenya \\ ${ }^{7}$ Moi University, School of Nursing, Department of Midwifery and Gender, Eldoret, Kenya \\ ${ }^{8}$ Department of Nursing, Kisii University College, Kenya \\ ${ }^{9}$ Indiana University School of Medicine, Indianapolis, Indiana, United States of America \\ ${ }^{10}$ Moi University, School of Medicine, Department of Internal Medicine, Eldoret, Kenya
}

\begin{abstract}
Introduction: The Moi Teaching and Referral Hospital and Moi University sponsored HIV/AIDs; AMPATH program cares for more than 65,000 adult HIV-infected patients whom $70 \%$ are women. $R H$ (Reproductive Health) and HIV clinics run vertically (independently), leading to increased number of hospital appointments and a reduced uptake of $R H$ services by these women. To respond, the program integrated $R H$ services into one of its HIV clinics.

Objectives: 1) To describe AMPATH's integration of RH services into HIV care, including: family planning $(F P)$ ), cervical cancer screening with acetic acid (VIA), antenatal care, and postnatal care; and 2) To describe the uptake of these services

Methods: A descriptive study of the integration between October 1st 2007 and May 31st 2009. HIV-infected women receive same-day 'one-stop-shop' care of RH and HIV care.

Results: Of 3,135 female clinic patients, 1,420 (45\%) received $R H$ services during this period: 1) All 1,420 were counseled for modern FP methods and screened for STIs, 2) 565 (40\%) were provided antenatal services, 3) $169(12 \%)$ were provided postnatal services, and 4) under a research protocol, 150 (11\%) were screened for cervical cancer.
\end{abstract}

Conclusion: Integration described here is a successful approach towards addressing RH needs faced by HIVinfected patients

Keywords: HIV care, Integration, Reproductive health services, AMPATH

\section{Introduction}

Sub-Saharan Africa carries a huge burden of the global HIV epidemic with nearly two-thirds of those living with HIV/AIDS residing in this region. $60 \%$ of people living with HIV/AIDS in Africa are women in their reproductive years ${ }^{1}$. This pattern is typified in Kenya, where $60 \%$ of the estimated 1.4 million adults who are HIV-infected are women of reproductive age ${ }^{2}$. Evidence from these countries reveal that combined antiretroviral therapy(cART) has dramatically improved the survival and quality of life for HIV-infected patients $^{3-5}$. Despite this fact, issues of fertility and childbearing have received relatively little attention ${ }^{6}$. HIVinfection modifies but does not eliminate reproductive desires, and intentions among HIV-infected patients. These patients continue to be sexually active and some have strong desires for biological children. On the other hand, some HIV-infected patients wish to avoid pregnancy due to fears of partner and infant infection, history of a previously HIV-infected baby and community disapproval associated with HIV and reproduction. Earlier research suggested that, in the absence of treatment, women who are HIV-infected were less sexually active compared to their uninfected counterparts, because of higher morbidity ${ }^{7,8}$. There is proof presently that, the success of prevention of mother-to-child transmission of HIV/AIDS (pMTCT) programs may alter attitudes in favor of childbearing ${ }^{6,9,10}$. In addition, clinical improvements and perceptions of reduced infectivity associated with the increased use of cART are anticipated to increase sexual activity among HIV-infected patients ${ }^{11,12}$. A study in South Africa, showed that fertility desire was associated with an increasing duration of cART among female participants ${ }^{6}$. 
HIV-infected women also have a much higher risk of human papillomavirus infection and consequently are at heightened risk for pre-invasive and invasive neoplasia of the cervix. During the preHAART era the lack of cervical cancer screening among HIV-infected women probably had little influence on their life expectancies because of the high competing mortality associated with HIV disease ${ }^{13,14}$ but this has changed in the HAART era. HIV/AIDS care and treatment programs provide an ideal platform to integrate cervical cancer prevention activities in resource constrain countries which face a dual burden of both HIV/AIDS and cervical cancer ${ }^{15}$. Limited access to cervical cancer prevention services currently is a counterforce to the life-prolonging potential of increased access to affordable antiretroviral therapy. Cervical cancer prevention strategies that use visual inspection with acetic acid (VIA) and same-visit cryotherapy ("see-and-treat") are costeffective alternatives to cytology-based screening programs. These procedures can be performed by nurses and other non-physician health-care workers and allow screening and treatment to be linked to the same clinic visit. In Zambia VIA-based prevention services that are nested within the context of antiretroviral therapy programs allow early detection of cervical cancer in high-risk HIV-infected women in a cost-effective way ${ }^{16}$.

In light of this considerable evidence, HIV-infected patients like their uninfected counterparts have reproductive needs and rights, the United States Agency for International Development-Academic Model for Providing Access To Healthcare (AMPATH) started a pilot module to integrate reproductive health services into its HIV care.

\section{Broad Aim}

To integrate reproductive health (RH) services into the AMPATH Eldoret module I HIV clinic.

\section{Specific Aims}

1) To integrate the following RH services into HIV care: counseling and provision of contraceptive services, screening for sexually transmitted infections (STI), cervical cancer screening with visual inspection with acetic acid (VIA) and Loop Electrosurgical Excision Procedure(LEEP), antenatal care, and postnatal care.

2) To describe the feasibility and uptake of these services among HIV-infected women.

\section{Study Design}

\section{Methods}

This was a clinic-based descriptive study of phase I of integrating RH services into HIV care. Results presented were collected during an 18 month pilot period of the study. The study was approved by the Indiana University School of Medicine Institutional Review Board and the Moi University School of Medicine Institutional Review and Ethics Committee. The pilot started in October 2007 and is still ongoing. The following RH services were integrated into HIV clinic: counseling and provision of contraceptive services, screening for STI, cervical cancer screening with visual inspection with acetic acid (VIA) and Loop Electrosurgical Excision Procedure (LEEP), antenatal care, and postnatal care.

\section{Study site and setting}

This study was carried out in the Eldoret module I clinic of the AMPATH program. The AMPATH program described elsewhere ${ }^{3,17-19}$ began to provide HIV care in 2001 and currently operates in 18 Kenya Ministry of Health facilities in western Kenya. As of May 2009, the program was caring for 86,193 HIVinfected patients across its 18 sites. Of these 70, 157 are adult patients of whom 48,549 (69.2\%) are female patients.

\section{Original AMPATH care model}

In the original AMPATH care model, the HIV clinic and the RH clinic were each vertically integrated and run independent of one another. The HIV clinic is run under the department of internal medicine and the RH clinic is run under the department of reproductive health. Patients who needed RH services (contraception, cancer of the cervix screening, STI screening, antenatal and postnatal care) were referred to the RH clinic after their appointment in the HIV clinic. In this model, it was the patients responsibly to ensure that they had their RH appointment after referral from the HIV clinic. Unlike HIV care which is provided free of charge, services in the RH clinic require patients to co-pay. The HIV care protocol has a well-structured outreach program for following loss to follow up patients, a luxury that is not available in the RH care protocol. Two challenges were observed based in this model of care. To start with, patients who managed to get RH clinic appointments had an increased burden of hospital visits. Secondly, there was a relative underutilization of RH services by HIVinfected patients due to the fact that getting an RH appointment depended solely on the individual patient and the RH care protocol was short of an outreach program analogous to the one in the HIV clinic. 


\section{Reproductive Health and HIV Care Integrated Model}

Integration of RH services into HIV care pilot study started in October of 2007 and is still ongoing in AMPATH's Eldoret module I clinic. In this model, RH services are housed within the HIV clinic and a hybrid of both vertical and horizontal integration is utilized. The vertical nature of both RH and HIV care is maintained to ensure that the focus and specific nature of these two service provisions is not weakened by a complete horizontal integration. The link between the two services forms the horizontal nature of the model and at module level both services are run under the same in charge. Services that are horizontally integrated are: the 'one-stopshop' nature of care, same-visit appointments, patient flow logistics, central check in and check out, use of same patient charts and filling, consultations, outreach services for loss to follow up patients, module progress meetings and passage of same messages on: adherence, importance of contraception, disclosure, and approach to future fertility desires. HIV-infected women receive both HIV care and RH services in the same module 'onestop-shop' care. The RH services offered are: counseling and provision of contraceptive methods, cancer of the cervix screening and LEEP, STI screening, antenatal care and postnatal care. Both HIV care and RH services are offered to HIV-infected women on the same-visit. After receiving HIV care patients are referred to the reproductive health room which is within the HIV clinic and forms part of patient flow in the clinic.

\section{Study Population}

HIV-infected women being cared for in the AMPATH Eldoret module I HIV clinic formed the study population. The specific eligibility criteria for: family planning was all HIV-infected women, antenatal care was pregnant patients, postnatal care was postnatal patients during their first postnatal clinic visit and cancer of the cervix screening was patients eligible for the cervical cancer screening study.

\section{Data Collection, Management and Analysis}

Five data collection forms are used in the integrated model, three exclusively designed for RH data collection (family planning/STI, antenatal and postnatal forms). The other two forms are the routine HIV care AMPATH adult initial and return visit forms. The RH forms collects information as described under protocols and form section. The routine HIV care adult encounter form collects data on patients HIV care and management. In addition to the forms the RH nurses keep diaries which are used to log in the RH services offered to patients. Data from all these forms are entered into the AMPATH Medical Records System (AMRS) which is a secure computerized database which uses an SQL platform. Data was extracted into SAS from the data base and manually from the diaries for this analysis.

\section{Results}

We were able to integrate RH services into the HIV clinic. Patients and healthcare providers liked the integrated model. Patients were able to get same-day services under the same roof. At the end of the 18 month pilot period, total of 3135 HIV-infected women were seen in the RH clinic. Of these, $1420(45 \%)$ were counseled and provided with modern family planning and STIs. There were 565(40\%) women who were provided antenatal services, and $169(12 \%)$ postnatal mothers were provided postnatal services. A further $150(11 \%)$ women were screened for cancer of the cervix and among these, 46(31\%) LEEP's were performed.

\section{Discussion}

Integration of RH services into HIV care is one of the feasible ways of ensuring that HIV-infected women get access to sexual and reproductive health services which is fundamental to the well-being of women. Integration such as described in this model is essential in ensuring that HIV-infected women get access to RH services: contraception, STI screening, antenatal care, postnatal care and cancer of the cervix screening. HIV is a special disease because of the stigma and labeling associated with it. Currently its chronic nature due to the positive effects of $\mathrm{cART}^{3-5}$ complicates it more by making its management infinite. HIV care is traditionally a vertical program due to the fact that it was an epidemic with high mortality rates and access to ARV's was a priority then. For this reason services that are not directly related to HIV disease like reproductive health services were not emphasized.

This study was not without challenges. The main challenge was having two traditionally vertical programs run under one roof and ensuring that the horizontal component functions well without erasing the important components of the vertical arm. We overcome this challenge by having the health care providers from both teams agree on what services to horizontally integrate coupled with an intensive sensitization and training 
of health care providers. The other challenge was a snail pace start at the beginning of the pilot which improved when the results of the integration began evident to the healthcare providers and patients.

\section{Conclusion and Recommendation}

From the study it came out clearly that HIV patients equally require reproductive health services. Thus provision of reproductive health services within an HIV care program as described in the study is a successful approach towards addressing reproductive health needs faced by HIV infected patients. The study also realized that both pregnancy and almost all HIV infections start with sex and until this intimate relationship between the two is realized, prevention of HIV will remain a mirage for a very long time. This integration is viable, feasible and cost-effective in resource limited settings like sub-Saharan where patients lack resources to attend all hospital appointments, which except for HIV care (offered free of charge) they are required to pay for services. We recommend that care programs and funding agencies should incorporated reproductive health services into HIV care.

\section{Acknowledgements}

- Moi Teaching and Referral Hospital, USAID-AMPATH Module I

- Moi University School of Medicine, Indiana University School of Medicine

This research has been supported by the President's Emergency Plan for AIDS Relief (PEPFAR) through USAID under the terms of Cooperative Agreement No. AID-623-A-12-0001 It is made possible through joint support of the United States Agency for International Development (USAID). The contents of this research are the sole responsibility of AMPATH and do not necessarily reflect the views of USAID or the United States Government.

[1]. UNAIDS. 2008 Report on the Global AIDS Epidemic. Geneva: Joint UN Program on HIV/AIDS (UNAIDS). 2008

[2]. MOH. Kenya AIDS Indicator Survey (KAIS) 2007; 2008.

[3]. Wools-Kaloustian K, Kimaiyo S, Diero L, et al. Viability and effectiveness of large-scale HIV treatment initiatives in sub-Saharan Africa: experience from western Kenya. Aids. Jan 2 2006; 20(1):41-48.

[4]. Marins JR, Jamal LF, Chen SY, et al. Dramatic improvement in survival among adult Brazilian AIDS patients. Aids. Jul 25 2003;17(11):1675-1682.

[5]. Seyler C, Anglaret X, Dakoury-Dogbo N, et al. Medium-term survival, morbidity and immunovirological evolution in HIV-infected adults receiving antiretroviral therapy, Abidjan, Cote d'Ivoire. Antivir Ther. Oct 2003;8(5):385-393.

[6]. Myer L, Morroni C, Rebe K. Prevalence and determinants of fertility intentions of HIV-infected women and men receiving antiretroviral therapy in South Africa. AIDS Patient Care STDs. Apr 2007; 21(4):278-285.

[7]. Maier M, Andia I, Emenyonu N, et al. Antiretroviral therapy is associated with increased fertility desire, but not pregnancy or live birth, among HIV+ women in an early HIV treatment program in rural Uganda. AIDS Behav. Jun 2009;13 Suppl 1:28-37.

[8]. Gray RH, Wawer MJ, Serwadda D, et al. Population-based study of fertility in women with HIV-1 infection in Uganda. Lancet. Jan 10 1998; 351(9096):98-103.

[9]. Cooper D, Moodley J, Zweigenthal V, Bekker LG, Shah I, Myer L. Fertility intentions and reproductive health care needs of peo ple living with HIV in Cape Town, South Africa: implications for integrating reproductive health and HIV care services. AIDS Behav. Jun 2009; 13 Suppl 1:38-46.

[10]. Cooper D, Harries J, Myer L, Orner P, Bracken H, Zweigenthal V. "Life is still going on": reproductive intentions among HIVpositive women and men in South Africa. Soc Sci Med. Jul 2007;65(2):274-283.

[11]. Kaida A, Andia I, Maier M, et al. The potential impact of antiretroviral therapy on fertility in sub-Saharan Africa. Curr HIV/AIDS Rep. Nov 2006; 3(4):187-194.

[12]. Kaida A, Gray G, Bastos FI, et al. The relationship between HAART use and sexual activity among HIV-positive women of reproductive age in Brazil, South Africa, and Uganda. AIDS Care. Jan 2008; 20(1):21-25.

[13]. Franceschi S, Jaffe H. Cervical cancer screening of women living with HIV infection: A must in the era of antiretroviral therapy. Clin Infect Dis. Aug 15 2007; 45(4):510-513.

[14]. Parham GP, Sahasrabuddhe VV, Mwanahamuntu MH, et al. Prevalence and predictors of squamous intraepithelial lesions of the cervix in HIV-infected women in Lusaka, Zambia. Gynecol Oncol. Dec 2006;103(3):1017-1022.

[15]. Stringer JS, Zulu I, Levy J, et al. Rapid scale-up of antiretroviral therapy at primary care sites in Zambia: feasibility and early outcomes. Jama. Aug 16 2006; 296(7):782-793.

[16]. Pfaendler KS, Mwanahamuntu MH, Sahasrabuddhe VV, Mudenda V, Stringer JS, Parham GP. Management of cryotherapyineligible women in a "screen-and-treat" cervical cancer prevention program targeting HIV-infected women in Zambia: lessons from the field. Gynecol Oncol. Sep 2008; 110(3):402-407.

[17]. Mamlin JK, SN; Nyandiko, Tierney, WM. Academic institutions linking access to treatment and prevention: Case study. World Health Organization 2004.

[18]. Inui TS, Nyandiko WM, Kimaiyo SN, et al. AMPATH: living proof that no one has to die from HIV. J Gen Intern Med. Dec 2007; 22(12):1745-1750.

[19]. Voelker R. Conquering HIV and stigma in Kenya. Jama. Jul 14 2004; 292(2):157-159. 